\title{
Synthesis of 7-substituted pyrazolo[1,5-a]pyrimidine-3- carboxamides as potential non benzodiazepine hypnotics
}

\author{
Haider Behbehani,* Hamada Mohamed Ibrahim, and Saad Makhseed \\ Chemistry Department; Faculty of Science; Kuwait University; P.O. Box 5969; \\ Safat; 13060-Kuwait \\ E-mail: hbehbehani@live.com
}

\begin{abstract}
A variety of new pyrazolo[1,5-a]pyrimidines has been prepared as potential drugs for the treatment of insomnia. The general synthetic route used for this purpose involves the condensation of substituted cyanoacetamides 5a-c, prepared by reaction of cyanoacetic acid with amines in presence of acetic anhydride, with dimethylformamide dimethylacetal and subsequent treatment of the formed enamines 6 with hydrazine hydrate. This process affords the corresponding aminopyrazole carboxamides $\mathbf{9}$ that react with the enaminonitrile 12 to generate the targets. Structures of the substance prepared in these sequences were established by using spectroscopic methods, including ${ }^{15} \mathrm{~N}$ HMBC and NOE difference experiments, as well as X-raycrystallographic analysis.
\end{abstract}

Keywords: Cyanoacetamides, aminopyrazoles, zaleplon analogs, pyrazolo[ $[1,5-a]$ pyrimidines, arylhydrazonoisoxazole

\section{Introduction}

Almost $40 \%$ of adults between ages $40-70$ suffer from insomnia at least one time during their lives. The drug Zaleplon $\mathbf{1}^{1-4}$ has been found to be efficient in the treatment of sleep disorder where difficulty in falling asleep is the primary issue. Unlike many other hypnotic drugs, this substance does not interfere with sleep architecture and can be administered for up to five weeks without the risk of dependence or rebound insomnia upon discontinuation. Indiplon $2^{1,5}$ has recently been released for use for the same purpose while the developments of ocinaplon $3{ }^{6}$ which is an anxiolytic drug in the pyrazolopyrimidine family of drugs, has been discontinued owing to liver complications observed in clinical trials. As a result, a need exists for the development of analogs of 1-3. In the studies described in this publication, a new, simple and efficient route to 7 -substituted pyrazolo[1,5-a]pyrimidine-3-carboxamides ${ }^{7-10}$ that have structures related to 1-3 has been developed. 

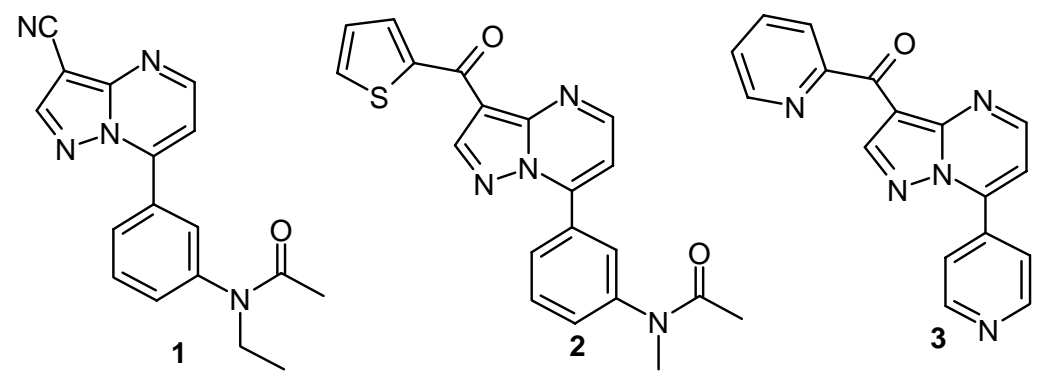

\section{Results and Discussion}

Preparation of the target compounds was initiated by reactions of amines 4a-c with mixtures of acetic anhydride and cyanoacetic acid, following the recently published procedure. ${ }^{7,11,12}$ This process affords the cyanoacetamides 5 a-c in $85-93 \%$ yields (Scheme 1). The products undergo reaction with dimethylformamide/dimethylacetal (DMF/DMA) to yield the corresponding enamines 6a-c. Although this process has the potential of producing mixtures of the stereoisomeric enamines 6 and 7, the fact that only the E-isomers 6 were generated has been demonstrated by using NOE experiments that showed that the vinyl protons were spatially proximate to the amide $\mathrm{NH}$.

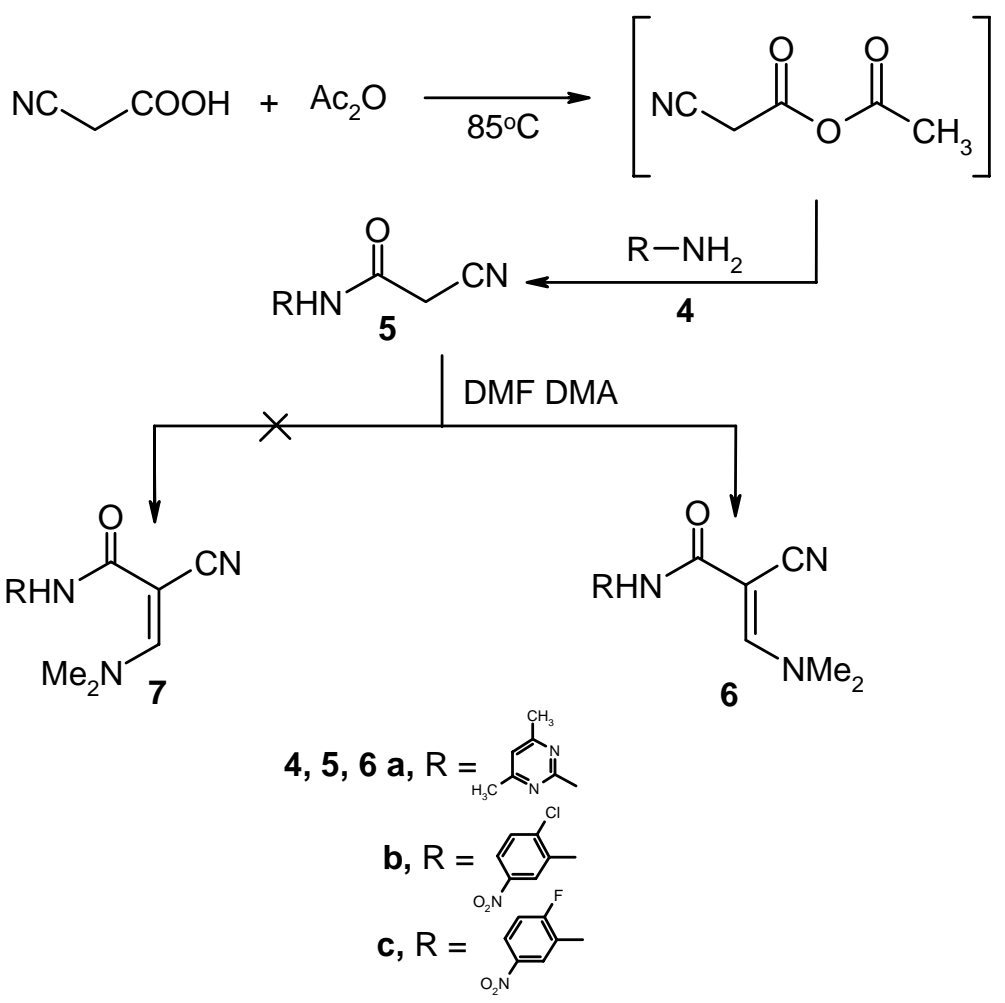

\section{Scheme 1}


An ethanol solution of the cyano-enamine $\mathbf{6 a}$ and hydrazine hydrate at room temperature undergoes reaction to form the acyclic hydrazine derivative 8 (Scheme 2). When 8 was either stirred at $50{ }^{\circ} \mathrm{C}$ or heated in pyridine solution, cyclization gradually took place via addition of hydrazine to the cyano group. This process generated pyrazole 9a, which was shown by using ${ }^{1} \mathrm{H}$ NMR spectroscopy (eg., ring $\mathrm{CH}$ at $\delta 8.1 \mathrm{ppm}$, singlet) to be the tautomeric form shown in Scheme 2. Stirring a solution of $\mathbf{6}$ in DMSO at reflux produces a mixture of $9 \mathbf{a}$ and the tautomer $\mathbf{1 0}$ in a 2 to 1 ratio. Unexpectedly, stirring a solution of $\mathbf{8}$ in ethanol afforded the cyanopyrazole 11. Consequently, it appeared that 9 was the kinetic product and $\mathbf{1 1}$ was the thermodynamic product. In contrast to $\mathbf{6 a}$, an ethanol solution of enamine $\mathbf{6 b}$ reacted with hydrazine hydrate at reflux to give the amino pyrazole $\mathbf{9 b}$ as a single product (Scheme 2).

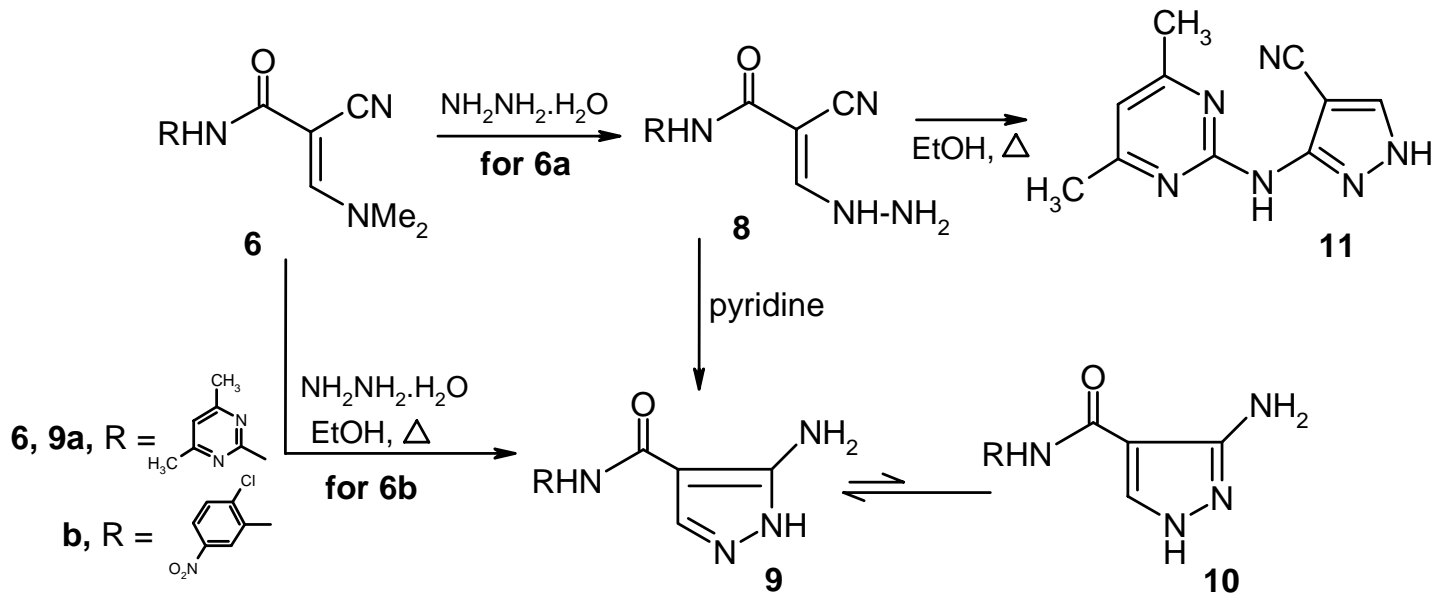

Scheme 2

Pyrazoles 9a,b reacted smoothly with enaminonitrile 12 in pyridine to yield the aminopyrazolo[1,5-a]pyrimidines $\mathbf{1 3}$ and not the regioisomeric compounds 14. Attempts to isolate the acyclic intermediate in these processes were not successful. ${ }^{15} \mathrm{~N}$ HMBC analysis was used to demonstrate that the structures of $\mathbf{1 3}$ were those of 7-aminopyrazolo[1,5-a]pyrimidines.

Specifically, the position of the amino group on C-7 was evidenced by ${ }^{4} \mathrm{~J}$ coupling of the protons at $\delta 8.4 \mathrm{ppm}$ with the $\mathrm{sp}^{3}$ bridged head nitrogen atom at $\delta 210.4 \mathrm{ppm}$. It is expected that if the regioisomeric structure $\mathbf{1 4}$ had been formed, protons of the amino group at $\delta 8.4 \mathrm{ppm}$ would have been coupled only with $\mathrm{N}-4$ at $\delta 224.2 \mathrm{ppm}$. In addition, 13 was observed to react with benzoyl chloride in pyridine to afford the benzoyl derivative $\mathbf{1 5}$.

The reaction of pyrazoles 9 with the enaminones 16 led to formation of the acyclic products 17 that underwent cyclization to generate 18 upon stirring in DMF containing sodium acetate at reflux. It should be noted that the pyrazolo[1,5-a]pyrimidines $\mathbf{1 8}$ could be prepared directly from $\mathbf{9}$ and 16 by stirring in refluxing pyridine overnight (Scheme 3 ). 


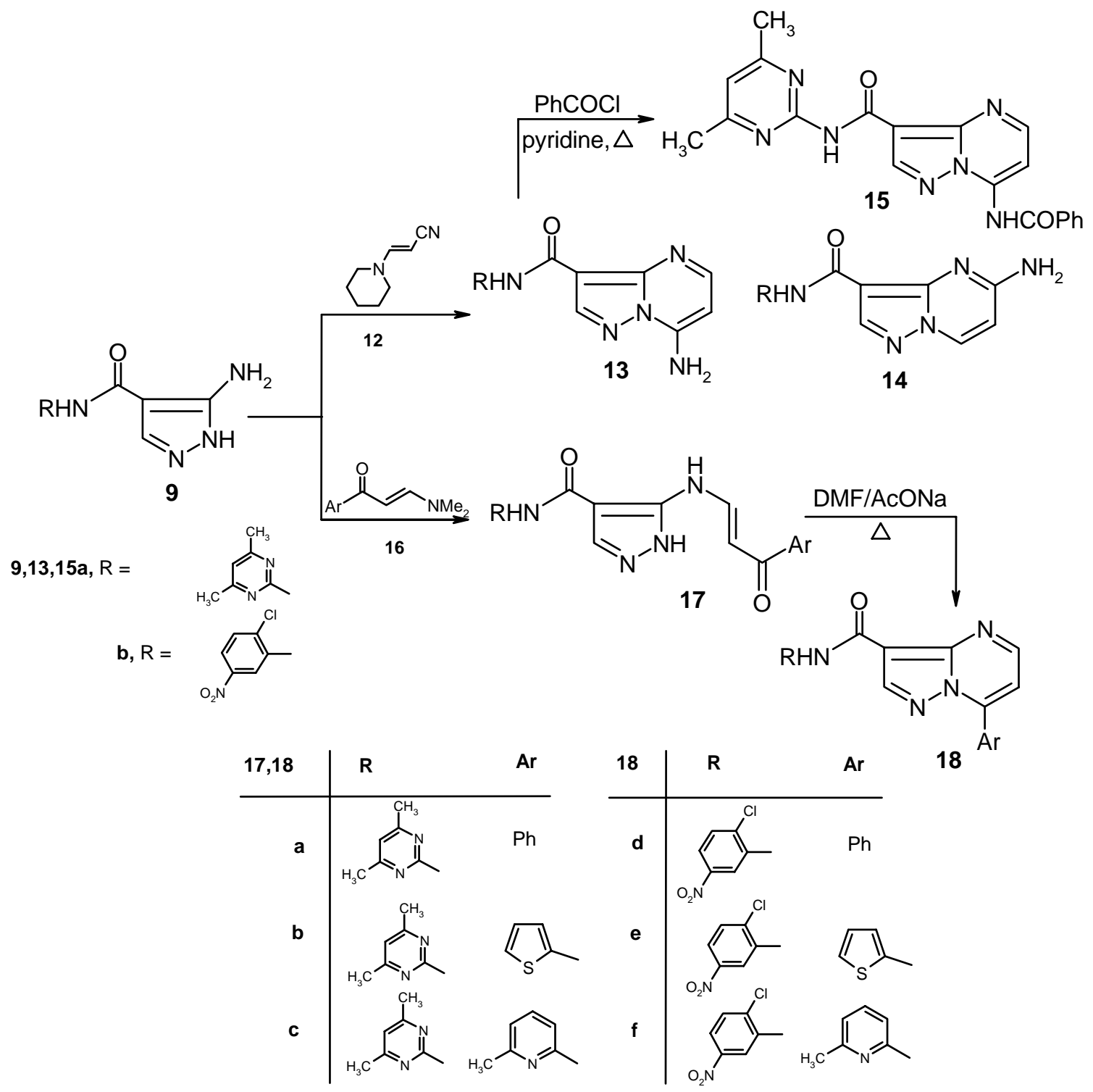

\section{Scheme 3}

Attempts to synthesize pyrazolo[3,4- $d]$ pyrimidines 20 by the reactions of 9a,b with dimethylformamide/dimethylacetal (DMF/DMA) failed and only the acyclic amidines 19 were formed. These substances did not cyclize to generate the corresponding pyrazolopyrimidines. Although, 19 may exist in 1H-3-amino forms, their existence in 1H-5-amino forms was established by analysis of ${ }^{1} \mathrm{H}$ NMR spectra that showed that $\mathrm{H}-3$ is a singlet at $\delta 9.27$. Reaction of 9b with excess dimethylformamide/dimethylacetal affords the hydrazone 21 as a result of methylation of the pyrazole NH (Scheme 4). 


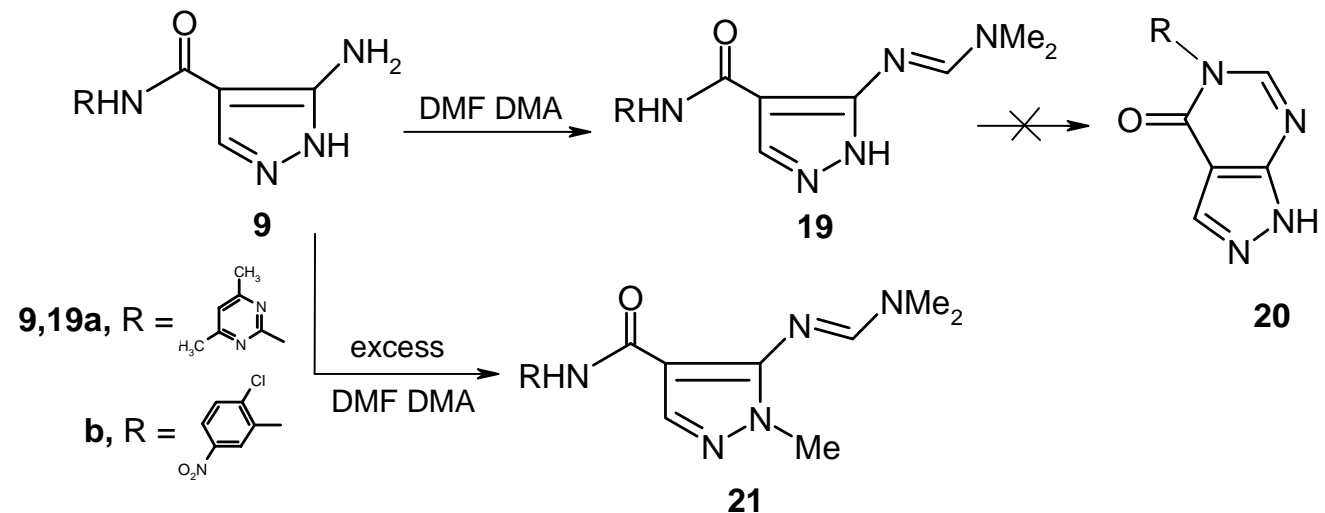

\section{Scheme 4}

Further investigations showed that 5a-c reacted with aryldiazonium chloride to yield arylhydrazones 22a-d whose structures were established by using X-ray crystallography (Figure 1). The previous finding that 2-arylhydrazono-3-oxoalkanenitriles prefer to exist in anti conformations (eg., 22) rather than syn. Hydrogen bonded forms provides further support for the conclusion that stereoelectronic factors are more important than hydrogen bonding interactions in governing conformations in these systems. ${ }^{13,14}$

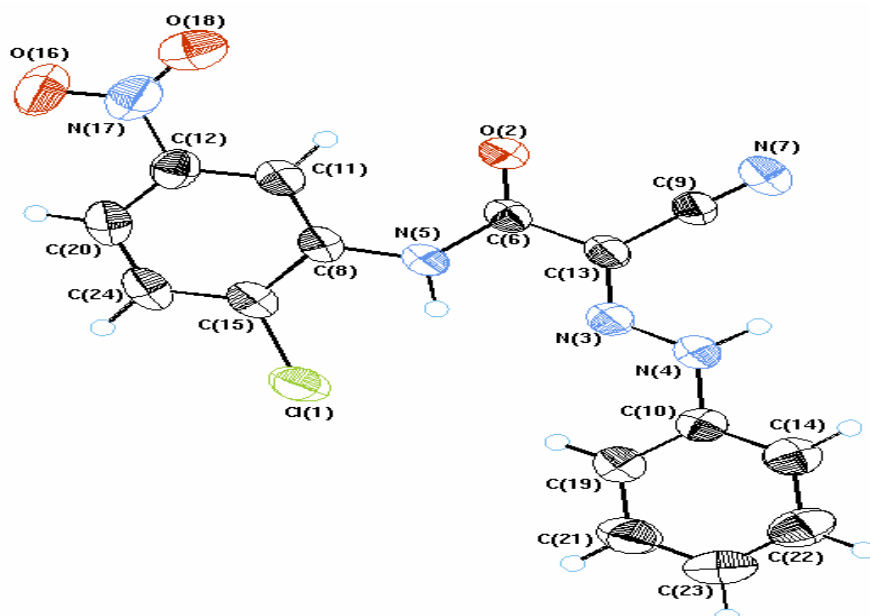

Figure 1. X-Ray crystallographic structure of 22c.

The results of previous studies ${ }^{7,15,16}$ demonstrated that the arylhydrazonitriles could be utilized as precursors for 1,2,3-triazoles, isoxazoles and pyrazoles. In the current effort, the possible utility of the arylhydrazones 22a-d as precursors for the above mentioned heterocycles was explored. Thus, reaction of 22c with hydroxylamine hydrochloride in refluxing DMF containing sodium acetate was observed to produce $\mathbf{2 4}$ via the amidoxime 23 . The structure of 24 was established on the basis of the results of NOE difference experiments. Specifically, irradiation of the amine hydrogen resonance at $\delta 6.21 \mathrm{ppm}$ did not enhance the aryl hydrogen 
signals at $\delta$ 7.41-8.91 $\mathrm{ppm}$. On the other hand, reaction of 22a with hydroxylamine hydrochloride in refluxing DMF in the presence of sodium acetate yielded the isoxazolone 25 via the amidoxime 23 through loss of an amine and not water. This same isooxazolone can be obtained by cyclization of the amidoxime 26, which was previously prepared by Elnagdi et al. ${ }^{17}$ by treatment with sodium ethoxide (Scheme 5).

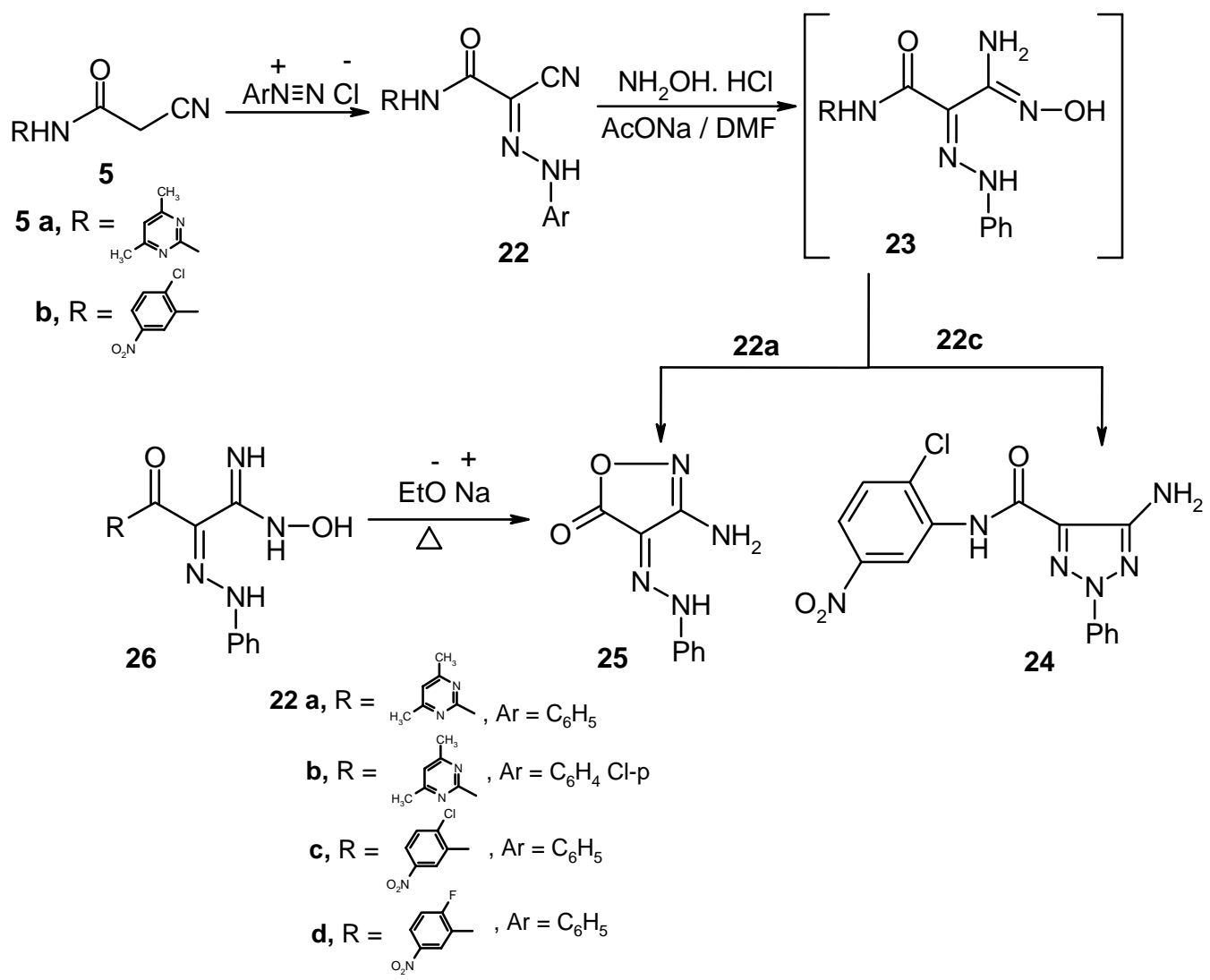

\section{Scheme 5}

\section{Conclusions}

The results of the study described above have led to the development of a simple approach for synthesis of 5-aminopyrazole-4-carboxamides and methodology for the conversion of these compounds into pyrazolo[1,5-a]pyrimidines, substances that potentially interesting biological and medicinal properties. Furthermore, the observations made during this work showed that the reaction of hydrazine hydrate with enamines can afford cyanopyrazoles or aminopyrazoles, depending on the reaction conditions used. 


\section{Experimental Section}

General. All melting points are reported uncorrected. IR spectra were recorded using $\mathrm{KBr}$ disks using a Perkin-Elmer System 2000 FT-IR spectrophotometer. ${ }^{1} \mathrm{H}$ NMR $(400 \mathrm{MHz})$ and ${ }^{13} \mathrm{C}$ NMR $(100 \mathrm{MHz})$ spectra were recorded on a Bruker DPX 400, $400 \mathrm{MHz}$ super-conducting NMR spectrometer on $\mathrm{CDCl}_{3}$ or DMSO- $d_{6}$ solutions with TMS as internal standard. Chemical shifts are reported in ppm. Mass spectra were measured using a VG Autospec Q MS 3 and MS 9 (AEI) spectrometers with EI $(70 \mathrm{EV})$. Microanalyses were performed on a LECO CHNS-932 Elemental Analyzer. The crystal structure was determined by using a X-ray instrument at the National Research Center, Dokki, Cairo.

\section{General procedure for the preparation of 5 a-c}

A solution of cyanoacetic acid $(10 \mathrm{mmol})$ in $\mathrm{Ac}_{2} \mathrm{O}(10 \mathrm{~mL})$ was heated on a water bath at $85{ }^{\circ} \mathrm{C}$ for $10 \mathrm{~min}$. Then, the appropriate starting materials $4 \mathbf{a}-\mathbf{c}$ were added to the reaction mixture and heating was continued at reflux for a further $15 \mathrm{~min}$. The reaction mixture was cooled and poured onto cold water. The solid products 5a-c were collected by filtration and crystallized from the appropriate solvent .

2-Cyano- $\mathrm{N}$-(4,6-dimethylpyrimidin-2-yl)acetamide (5a). Recrystallized from acetic acid as creamy white crystals, $1.6 \mathrm{~g}(85 \%)$, mp $130{ }^{\circ} \mathrm{C}$; IR (KBr): $3221(\mathrm{NH}), 2259(\mathrm{CN}), 1680 \mathrm{~cm}^{-1}$ (CO); ${ }^{1} \mathrm{H}$ NMR $\left(\mathrm{CDCl}_{3}\right): \delta 2.47\left(\mathrm{~s}, 6 \mathrm{H}, 2 \mathrm{CH}_{3}\right), 4.30\left(\mathrm{~s}, 2 \mathrm{H}, \mathrm{CH}_{2}\right), 6.82(\mathrm{~s}, 1 \mathrm{H}$, pyrimidine $\mathrm{H}-5)$ and $9.74 \mathrm{ppm}(\mathrm{s}, 1 \mathrm{H}, \mathrm{NH}) ;{ }^{13} \mathrm{C}$ NMR $\left(\mathrm{CDCl}_{3}\right): \delta 23.73\left(2 \mathrm{CH}_{3}\right), 28.88\left(\mathrm{CH}_{2}\right), 114.13(\mathrm{CN})$, 116.12 (pyrimidine C-5), 156.23, 163.70 (Ar-C) and 168.75 ppm (CO); MS (EI): m/z (\%) 192 $\left(\mathrm{M}^{+}+1,10.35\right), 190\left(\mathrm{M}^{+}, 42.80\right), 123$ (100), 107 (18.25), 96 (23). Anal. Calcd. for $\mathrm{C}_{9} \mathrm{H}_{10} \mathrm{~N}_{4} \mathrm{O}$ (190.21): C, 56.83; H, 5.30; N, 29.46. Found: C, 56.68; H, 5.42; N, 29.32.

$\mathrm{N}$-(2-Chloro-5-nitrophenyl)-2-cyanoacetamide (5b). Recrystallized from EtOH as creamy white crystals, $2.2 \mathrm{~g}(93 \%), \mathrm{mp} 199-201{ }^{\circ} \mathrm{C}$; IR (KBr): $3292(\mathrm{NH}), 2256(\mathrm{CN}), 1677 \mathrm{~cm}^{-1}(\mathrm{CO})$; ${ }^{1} \mathrm{H}$ NMR (DMSO-d6): $\delta 4.10$ (s, 2H, CH $\left.\mathrm{CH}_{2}\right), 7.82(\mathrm{~d}, J=8.0 \mathrm{~Hz}, 1 \mathrm{H}, \mathrm{Ar}-\mathrm{H}$ ), $8.05(\mathrm{~d}, J=8.0 \mathrm{~Hz}$, $1 \mathrm{H}, \mathrm{Ar}-\mathrm{H}$ ), $8.70(\mathrm{~s}, 1 \mathrm{H}, \mathrm{Ar}-\mathrm{H})$ and $10.30 \mathrm{ppm}(\mathrm{s}, 1 \mathrm{H}, \mathrm{NH}) ;{ }^{13} \mathrm{C}$ NMR (DMSO-d6): $\delta 26.62$ $\left(\mathrm{CH}_{2}\right), 115.64(\mathrm{CN}), 119.49(\mathrm{CH}), 120.90(\mathrm{CH}), 130.90(\mathrm{CH}), 132.21(\mathrm{C}), 135.27(\mathrm{C}), 146.30(\mathrm{C})$ and $162.68 \mathrm{ppm}(\mathrm{CO})$; MS (EI): m/z (\%) $240\left(\mathrm{M}^{+}+1,12.9\right), 239\left(\mathrm{M}^{+}, 31.3\right), 204$ (100), 172 (58.5), 126 (32.8). Anal. Calcd. for $\mathrm{C}_{9} \mathrm{H}_{6} \mathrm{ClN}_{3} \mathrm{O}_{3}$ (239.62): C, 45.11; H, 2.52; Cl, 14.80; N, 17.54. Found: $\mathrm{C}, 45.22 ; \mathrm{H}, 2.46 ; \mathrm{Cl}, 14.93 ; \mathrm{N}, 17.59$.

2-Cyano- $\mathrm{N}$-(2-fluoro-5-nitrophenyl) acetamide (5c). Recrystallized from $\mathrm{EtOH}$ as white crystals, $1.9 \mathrm{~g}(89 \%), \mathrm{mp} 168-170{ }^{\circ} \mathrm{C}$; IR (KBr): $3320(\mathrm{NH}), 2264(\mathrm{CN}), 1708 \mathrm{~cm}^{-1}(\mathrm{CO}) ;{ }^{1} \mathrm{H}$ NMR (DMSO-d6): $\delta 4.07$ (s, 2H, CH 2 ), 7.59-8.94 (m, 3H, Ar-H ), $10.61 \mathrm{ppm}(\mathrm{s}, 1 \mathrm{H}, \mathrm{NH}) ;{ }^{13} \mathrm{C}$ NMR (DMSO-d6): $\delta$ 26.80( $\left(\mathrm{CH}_{2}\right), 115.61(\mathrm{CN}), 116.86,117.95,121.00,126.71,143.77,155.00$, (Ar-C) and $162.66 \mathrm{ppm}(\mathrm{CO}) ; \mathrm{MS}(\mathrm{EI}): \mathrm{m} / \mathrm{z}(\%) 224\left(\mathrm{M}^{+}+1,12.5\right), 223\left(\mathrm{M}^{+}, 73\right), 156(100), 110$ (58.6). Anal. Calcd. for $\mathrm{C}_{9} \mathrm{H}_{6} \mathrm{FN}_{3} \mathrm{O}_{3}$ (223.16): C, 48.44; H, 2.71; N, 18.83. Found: C, 48.26; H, $2.85 ; \mathrm{N}, 18.79$. 


\section{General procedure for the preparation of 6a-c}

A mixture of 5a-c (10 mmol) and DMFDMA (1.2 g, $10 \mathrm{mmol})$ in EtOH $(25 \mathrm{~mL})$ was stirred at room temperature for $4 \mathrm{~h}$ (for6a) or at reflux for $2 \mathrm{~h}$ (for $\mathbf{6 b}$ and $\mathbf{6 c}$ ). The separated solid products formed on standing at room temperature were collected by filtration, washed by EtOH and rcrystallized from the appropriate solvent.

(E)-2-Cyano-3-(dimethylamino)- $\boldsymbol{N}$-(4,6-dimethylpyrimidin-2-yl)acrylamide (6a). Recrystall -ized from EtOH as creamy white crystals, $1.85 \mathrm{~g}(76 \%), \mathrm{mp} 164{ }^{\circ} \mathrm{C}$; IR (KBr): $3399(\mathrm{NH})$, $2194(\mathrm{CN}), 1691 \mathrm{~cm}^{-1}(\mathrm{CO}) .{ }^{1} \mathrm{H}$ NMR $\left(\mathrm{CDCl}_{3}\right): \delta 2.44\left(\mathrm{~s}, 6 \mathrm{H}, 2 \mathrm{CH}_{3}\right), 3.26\left(\mathrm{~s}, 3 \mathrm{H}, \mathrm{CH}_{3}\right), 3.40(\mathrm{~s}$, $\left.3 \mathrm{H}, \mathrm{CH}_{3}\right), 6.75(\mathrm{~s}, 1 \mathrm{H}$, pyrimidine $\mathrm{H}-5), 7.93(\mathrm{~s}, 1 \mathrm{H}$, olefinic $\mathrm{CH})$ and $8.28 \mathrm{ppm}(\mathrm{s}, 1 \mathrm{H}, \mathrm{NH}) ;{ }^{13} \mathrm{C}$ NMR $\left(\mathrm{CDCl}_{3}\right): \delta$ 23.93 $\left(2 \mathrm{CH}_{3}\right), 38.63\left(\mathrm{CH}_{3}\right), 47.81\left(\mathrm{CH}_{3}\right), 71.64(\mathrm{C}-2), 115.60$ (pyrimidine $\left.\mathrm{C}-5\right)$, $119.24(\mathrm{CN}), 157.28(\mathrm{C}), 157.43$ (olefinic $\mathrm{CH}), 162.63(\mathrm{C})$ and $168.13 \mathrm{ppm}(\mathrm{CO}) ; \mathrm{MS}(\mathrm{EI}): \mathrm{m} / \mathrm{z}$ (\%) $246\left(\mathrm{M}^{+}+1,8.24\right), 245\left(\mathrm{M}^{+}, 41.5\right), 150(24.7), 123$ (100). Anal. Calcd. for $\mathrm{C}_{12} \mathrm{H}_{15} \mathrm{~N}_{5} \mathrm{O}$ (245.29): C, 58.76; H, 6.16; N, 28.55; Found: C, 58.65; H, 6.23; N, 28.59.

(E)-N-(2-Chloro-5-nitrophenyl)-2-cyano-3-(dimethylamino)acrylamide (6b). Recrystallized from EtOH/DMF as buff crystals, $2.45 \mathrm{~g}(83 \%), \mathrm{mp} 236{ }^{\circ} \mathrm{C}$; IR (KBr): 3391, (NH), $2183(\mathrm{CN})$, $1687 \mathrm{~cm}^{-1}(\mathrm{CO}) .{ }^{1} \mathrm{H} \mathrm{NMR}\left(\mathrm{CDCl}_{3}\right): \delta 3.31\left(\mathrm{~s}, 3 \mathrm{H}, \mathrm{CH}_{3}\right), 3.44\left(\mathrm{~s}, 3 \mathrm{H}, \mathrm{CH}_{3}\right), 7.55(\mathrm{~d}, J=8.6 \mathrm{~Hz}$, $1 \mathrm{H}, \mathrm{Ar}-\mathrm{H}$ ), 7.89 (d, $J=8.6 \mathrm{~Hz}, 1 \mathrm{H}, \mathrm{Ar}-\mathrm{H}), 7.92(\mathrm{~s}, 1 \mathrm{H}$, olefinic $\mathrm{CH}), 8.43$ (br, $1 \mathrm{H}, \mathrm{NH})$ and 9.37 ppm (s, 1H, Ar-H); ${ }^{13} \mathrm{C}$ NMR $\left(\mathrm{CDCl}_{3}\right): \delta 38.81\left(\mathrm{CH}_{3}\right), 47.91\left(\mathrm{CH}_{3}\right), 71.30(\mathrm{C}-2), 115.80$, 118.42, $118.97(\mathrm{CN}), 128.98,129.49,136.04,147.08,157.00$ and $163.25 \mathrm{ppm}(\mathrm{CO})$; MS (EI): $\mathrm{m} / \mathrm{z}(\%) 295\left(\mathrm{M}^{+}+1,4.1\right), 294\left(\mathrm{M}^{+}, 12.2\right), 123$ (100). Anal. Calcd. for $\mathrm{C}_{12} \mathrm{H}_{11} \mathrm{ClN}_{4} \mathrm{O}_{3}$ (294.70): C, 48.91; H, 3.76; Cl, 12.03; N, 19.01. Found: C, 49.02; H, 3.66; Cl, 11.89; N, 19.15.

(E)-2-Cyano-3-(dimethylamino)-N--(2-fluoro-5-nitrophenyl)acrylamide (6c). Recrystallized from EtOH/dioxane as pale yellow crystals, $2.1 \mathrm{~g}(75 \%), \mathrm{mp} 148-150{ }^{\circ} \mathrm{C}$; IR (KBr): $3259(\mathrm{NH})$, $2186(\mathrm{CN}), 1679 \mathrm{~cm}^{-1}(\mathrm{CO}) .{ }^{1} \mathrm{H}$ NMR (DMSO-d $\left.)_{6}\right): \delta 3.25\left(\mathrm{~s}, 3 \mathrm{H}, \mathrm{CH}_{3}\right), 3.31\left(\mathrm{~s}, 3 \mathrm{H}, \mathrm{CH}_{3}\right), 7.54-$ 8.7 (m, 3H, Ar-H ),7.88 (s, 1H, olefinic CH) and 9.1 ppm (br, 1H, NH); MS (EI): m/z (\%) 279 $\left(\mathrm{M}^{+}+1,4.3\right), 278\left(\mathrm{M}^{+}, 19.5\right), 123$ (100). Anal. Calcd. for $\mathrm{C}_{12} \mathrm{H}_{11} \mathrm{FN}_{4} \mathrm{O}_{3}$ (278.24): C, 51.80; $\mathrm{H}$, 3.98; N, 20.14. Found: C, 51.92; H, 4.06; N, 20.30.

(E)-2-Cyano- $\boldsymbol{N}$-(4,6-dimethylpyrimidin-2-yl)-3-hydrazinylacrylamide (8). A solution of the enaminonitrile 6a $(2.45 \mathrm{~g}, 10 \mathrm{mmol})$ and hydrazine hydrate $(80 \%, 0.65 \mathrm{ml})$ in EtOH $(50 \mathrm{~mL})$ stirred at reflux for 10 minand then cooled to room temperature Theformed solid was separated by filtration, washed with $\mathrm{EtOH}$, and crystallized from the EtOH/dioxan mixture as white crystals, $1.85 \mathrm{~g}(80 \%)$, mp $147{ }^{\circ} \mathrm{C}$; IR (KBr): 3404, 3296, 3221, $3189\left(\mathrm{NH}_{2}\right.$ and $\left.2 \mathrm{NH}\right), 2198$ (CN), $1690 \mathrm{~cm}^{-1}$ (CO). ${ }^{1} \mathrm{H}$ NMR (DMSO-d $): \delta 2.32\left(\mathrm{~s}, 6 \mathrm{H}, 2 \mathrm{CH}_{3}\right), 5.11\left(\mathrm{br}, 2 \mathrm{H}, \mathrm{NH}_{2}\right), 6.87(\mathrm{~s}$, $1 \mathrm{H}$, pyrimidine $\mathrm{H}-5), 7.72$ (s, 1H, olefinic $\mathrm{CH}), 8.89$ (br, $1 \mathrm{H}, \mathrm{NH})$ and $9.61 \mathrm{ppm}(\mathrm{br}, 1 \mathrm{H}, \mathrm{NH})$; ${ }^{13} \mathrm{C}$ NMR (pyridine-d5): $\delta 23.28\left(2 \mathrm{CH}_{3}\right), 66.82,109.29,114.64(\mathrm{CN}), 154.68,158.65,163.55$, 167.32, and $167.57 \mathrm{ppm}$ (carbons and CO); MS (EI): m/z (\%) $233\left(\mathrm{M}^{+}+1,21.8\right), 232\left(\mathrm{M}^{+}\right.$, 100), 124 (85.9), 123 (4835), 110 (90.3). Anal. Calcd. for $\mathrm{C}_{10} \mathrm{H}_{12} \mathrm{~N}_{6} \mathrm{O}$ (232.25): C, 51.72; H, 5.21; N, 36.19; Found: C, 51.64; H, 5.32; N, 36.24.

5-Amino- $N$-(4,6-dimethylpyrimidin-2-yl)-1H-pyrazole-4-carboxamide (9a). Method A. The crude reaction mixture of $\mathbf{8}$, formed in the manner described above, was stirred at $50{ }^{\circ} \mathrm{C}$ for $4 \mathrm{~h}$. 
Cooling to room temperature gave a precipitate which was separated by filtration, washed with EtOH and crystallized from EtOH/DMF to give white crystals.

Method B. A solution of 8 (1.1 g, $5 \mathrm{mmol})$ in pyridine $(20 \mathrm{~mL})$ was stirred at reflux for $3 \mathrm{~h}$ and concentrated in vacuo to give a residue which was triturated with EtOH to afford solid. Separation by filtration gave a solid that was washed with EtOH and crystallized from EtOH/DMF to give white crystals, $0.85 \mathrm{~g}(73 \%), \operatorname{mp} 256{ }^{\circ} \mathrm{C}$; IR ( $\left.\mathrm{KBr}\right): 3454,3343,3273,3129$ $\left(\mathrm{NH}_{2}\right.$ and $\left.2 \mathrm{NH}\right), 1663 \mathrm{~cm}^{-1}(\mathrm{CO}) .{ }^{1} \mathrm{H} \mathrm{NMR}\left(\mathrm{DMSO}-d_{6}\right): \delta 2.34\left(\mathrm{~s}, 6 \mathrm{H}, 2 \mathrm{CH}_{3}\right), 5.95(\mathrm{br}, 2 \mathrm{H}$, $\left.\mathrm{NH}_{2}\right), 6.89(\mathrm{~s}, 1 \mathrm{H}$, pyrimidine $\mathrm{H}-5), 8.10(\mathrm{~s}, 1 \mathrm{H}$, pyrazole $\mathrm{H}-3), 10.12(\mathrm{~s}, 1 \mathrm{H}, \mathrm{NH})$, and 11.87 ppm (br, 1H, NH); ${ }^{13} \mathrm{C}$ NMR (DMSO-d $): \delta 23.87\left(2 \mathrm{CH}_{3}\right), 98.15,110.07,116.11,138.88$, 157.99, 163.13 and $168.49 \mathrm{ppm}$ (carbons and CO); MS (EI): m/z (\%) $233\left(\mathrm{M}^{+}+1,15.6\right), 232$ $\left(\mathrm{M}^{+}, 100\right), 124$ (72), 123 (63), 110 (68.7). Anal. Calcd. for $\mathrm{C}_{10} \mathrm{H}_{12} \mathrm{~N}_{6} \mathrm{O}$ (232.25): C, 51.72; H, 5.21; N, 36.19; Found: C, 51.89; H, 5.36; N, 36.09.

\section{General procedure for the preparation of $9 b$ and 11}

A mixture of the enaminonitrile 6a $(2.45 \mathrm{~g}, 10 \mathrm{mmol})$ and hydrazine hydrate $(80 \%, 0.65 \mathrm{ml})$ in $\mathrm{EtOH}(40 \mathrm{~mL})$ was stirred at reflux for $2 \mathrm{~h}$, concentrated in vacuo to one third of its volume, and cooled to room temperature. The crude solid which formed was collected by filtration, washed with water, and crystallized from the appropriate solvent.

5-Amino- $\boldsymbol{N}$-(2-chloro-5-nitrophenyl)-1H-pyrazole-4-carboxamide (9b). Recrystallized from EtOH, $2.1 \mathrm{~g}$ (74 \%), mp 229 oC; IR (KBr): 3442, 3404, 3347, 3256 (NH2 and 2NH), $1673 \mathrm{~cm}-1$ (CO). 1H NMR (DMSO-d6): $\delta 6.08$ (br, 2H, NH2), 7.81 (d, J = 8.6 Hz, 1H, Ar-H ), 8.0-8.03 (m, 2H,1 Ar-H and pyrazole H-3), 8.65 (s, 1H, Ar-H), 9.44 (br, 1H, NH) and 11.97 ppm (br, 1H, NH); 13C NMR (DMSO-d6): $\delta$ 95.39, 120.08, 120.41, 130.60, 133.54, 136.28, 137.90, 146.21, 151.58 and $162.75 \mathrm{ppm}$ (Ar-carbons and CO); MS (EI): m/z (\%) $282(\mathrm{M}++1,6.5), 281(\mathrm{M}+, 18)$, 110 (100). Anal. Calcd. for $\mathrm{C}_{10} \mathrm{H}_{8} \mathrm{ClN}_{5} \mathrm{O}_{3}$ (281.66): C, 42.64; H, 2.86; Cl, 12.59; N, 24.86; Found: C, 42.55; H, 2.92; Cl, 12.48; N, 24.89.

3-(4,6-Dimethylpyrimidin-2-ylamino)-1H-pyrazole-4-carbonitrile (11). Recrystallized from EtOH/dioxan, $1.75 \mathrm{~g}(82 \%), \mathrm{mp} 262-264{ }^{\circ} \mathrm{C}$; IR (KBr): 3273, $3162(2 \mathrm{NH}), 2224(\mathrm{CN}), 1629 \mathrm{~cm}^{-}$ ${ }^{1}(\mathrm{C}=\mathrm{N}) .{ }^{1} \mathrm{H}$ NMR $\left(\mathrm{DMSO}-d_{6}\right): \delta 2.29\left(\mathrm{~s}, 6 \mathrm{H}, 2 \mathrm{CH}_{3}\right), 6.65(\mathrm{~s}, 1 \mathrm{H}$, pyrimidine $\mathrm{H}-5), 8.42(\mathrm{br}, 1 \mathrm{H}$, $\mathrm{NH}), 9.63$ (s, 1H, pyrazolyl H) and $13.16 \mathrm{ppm}(\mathrm{br}, 1 \mathrm{H}, \mathrm{NH}) ;{ }^{13} \mathrm{C}$ NMR (DMSO-d 6 ): $\delta 23.25$ $\left(2 \mathrm{CH}_{3}\right), 86.11,111.74,114.31,135.76,149.25,159.27$ and $167.40 \mathrm{ppm}$ (carbons and $\left.\mathrm{CO}\right)$; MS (EI): $\mathrm{m} / \mathrm{z}(\%) 215\left(\mathrm{M}^{+}+1,27.5\right), 214\left(\mathrm{M}^{+}, 95\right), 213$ (100). Anal. Calcd. for $\mathrm{C}_{10} \mathrm{H}_{10} \mathrm{~N}_{6}(214.23)$ : C, 56.07; H, 4.71; N, 39.23; Found: C, 55.93; H, 4.82; N, 39.19.

\section{General procedure for the preparation of 13a,b.}

Mixtures of 9a,b

( $5 \mathrm{mmol})$, and enamine $12(0.7 \mathrm{~g}, 5 \mathrm{mmol})$ in pyridine $(20 \mathrm{~mL})$ were stirred at reflux for $12 \mathrm{~h}$, cooled to room temperature, and poured into ice cold water. The aqueous solution was acidified with hydrochloric acid $(2 \mathrm{~N})$, forming a solid that was collected by filtration, washed with water and crystallized from the appropriate solvent. 
7-Amino- $\mathrm{N}$-(4,6-dimethylpyrimidin-2-yl)pyrazolo[1,5-a]pyrimidine-3-carboxamide (13a). Recrystallized from EtOH/DMF as buff crystals, $1.0 \mathrm{~g}(71 \%), \mathrm{mp}$ above $300{ }^{\circ} \mathrm{C}$; IR (KBr): 3436, 3337, $3171\left(\mathrm{NH}_{2}\right.$ and $\left.\mathrm{NH}\right), 1682(\mathrm{CO}), 1656 \mathrm{~cm}^{-1}(\mathrm{C}=\mathrm{N}) ;{ }^{1} \mathrm{H}$ NMR (DMSO-d $): \delta 2.37(\mathrm{~s}$, $\left.6 \mathrm{H}, 2 \mathrm{CH}_{3}\right), 6.34(\mathrm{~d}, J=5.6 \mathrm{~Hz}, 1 \mathrm{H}, \mathrm{H}-6), 6.93$ (s, $1 \mathrm{H}$, pyrimidine $\left.\mathrm{H}-5\right), 8.35$ (d, $1 \mathrm{H}, J=5.6 \mathrm{~Hz}$, $\mathrm{H}-5), 8.39$ (br, 2H, $\left.\mathrm{NH}_{2}\right), 8.54$ (s, $1 \mathrm{H}$, pyrazole $\left.\mathrm{H}-2\right)$ and $10.70 \mathrm{ppm}(\mathrm{s}, 1 \mathrm{H}, \mathrm{NH}) ;{ }^{13} \mathrm{C} \mathrm{NMR}$ $\left(\mathrm{DMSO}-d_{6}\right): \delta 23.41\left(2 \mathrm{CH}_{3}\right), 90.60,102.81,115.18,1425.27,147.00,149.09,151.21,157.22$, 158.50, 167.66 (Ar-C and CO); ${ }^{15} \mathrm{~N}$ HMBC (DMSO- $d_{6}$ ): showed $6 \delta$ at 79.4, 138.7, 210.4, 224.2, 260.3 and 265; MS (EI): m/z (\%) $284\left(\mathrm{M}^{+}+1,5.6\right), 283\left(\mathrm{M}^{+}, 23.5\right), 161$ (100), 123 (7.5). Anal. Calcd. for $\mathrm{C}_{13} \mathrm{H}_{13} \mathrm{~N}_{7} \mathrm{O}$ (283.29): C, 55.12; H, 4.63; N, 34.61. Found: C, 54.99; H, 4.69; N, 34.55. 7-Amino- $N$-(2-chloro-5-nitrophenyl)-pyrazolo[1,5-a]pyrimidine-3-carboxamide (13b). Recrystallized from DMF as yellow crystals, $1.25 \mathrm{~g}(75 \%)$, mp above $300{ }^{\circ} \mathrm{C}$; IR (KBr): $3409,3269,3201\left(\mathrm{NH}_{2}\right.$ and $\left.\mathrm{NH}\right)$, $1685(\mathrm{CO}), 1615 \mathrm{~cm}^{-1}(\mathrm{C}=\mathrm{N}) ;{ }^{1} \mathrm{H}$ NMR (DMSO- $\left.d_{6}\right): \delta 6.39(\mathrm{~d}, J=5.4 \mathrm{~Hz}, 1 \mathrm{H}, \mathrm{H}-6), 7.78(\mathrm{~d}, J=$ $9.0 \mathrm{~Hz}, 1 \mathrm{H}, \mathrm{Ar}-\mathrm{H}$ ), 7.89 (d, J=9.0 Hz, 1H, Ar-H ), 8.09 (br, 2H, NH 2$), 8.33$ (d, J=5.4 Hz, 1H, $\mathrm{H}-5), 8.55$ (s, 1H, Ar-H), 9.46 (s, 1H, pyrazole H-2) and 10.92 (s, 1H, NH); ${ }^{3} \mathrm{C}$ NMR (DMSO$\left.d_{6}\right): \delta 91.01,102.08,114.64,118.06,128.07,130.38,136.78,145.20,146.51,147.37,149.20$, 151.36, 160.66 (Ar-C and CO); MS (EI): m/z (\%) $333\left(\mathrm{M}^{+}+1,2.75\right), 332\left(\mathrm{M}^{+}, 8.3\right), 161$ (100), 133 (3.5). Anal. Calcd. for $\mathrm{C}_{13} \mathrm{H}_{9} \mathrm{ClN}_{6} \mathrm{O}_{3}$ (332.71): C, 46.93; H, 2.73; Cl, 10.66; N, 25.26. Found: C, 47.04; H, 2.79; Cl, 10.80; N, 25.32.

7-Benzamido- $N$-(4,6-dimethylpyrimidin-2-yl)pyrazolo[1,5-a]pyrimidine-3-carboxamide (15). A mixture of 13a $(0.7 \mathrm{~g}, 2.5 \mathrm{mmol})$, and benzoylchloride $(0.4 \mathrm{~g}, 2.5 \mathrm{mmol})$ in pyridine $(20 \mathrm{~mL})$ was stirred at reflux for 3 $\mathrm{h}$, cooled to room temperature and poured into ice cold water. The aqueous solution was acidified with hydrochloric acid $(2 \mathrm{~N})$, forming a solid that was collected by filtration, washed with water and crystallized from EtOH/DMF to give pale brown crystals, $0.67 \mathrm{~g}(70 \%), 300{ }^{\circ} \mathrm{C}$; IR (KBr): 3368, $3281(2 \mathrm{NH}), 1707,1623 \mathrm{~cm}^{-1}$ (2 CO); ${ }^{1} \mathrm{H}$ NMR (DMSO-d $\left.)_{6}\right): \delta 2.41(\mathrm{~s}, 6 \mathrm{H}$, $\left.2 \mathrm{CH}_{3}\right), 6.99$ (s, $1 \mathrm{H}$, pyrimidine H-5), 7.62 (t, $\left.J=8.0 \mathrm{~Hz}, 2 \mathrm{H}, \mathrm{Ar}-\mathrm{H}\right), 7.71$ (t, $J=8.0 \mathrm{~Hz}, 1 \mathrm{H}$, ArH), 7.96 (d, $J=5.0 \mathrm{~Hz}, 1 \mathrm{H}, \mathrm{H}-6), 8.06$ (d, $J=8.0 \mathrm{~Hz}, 2 \mathrm{H}$, Ar-H), 8.76 (s, 1H, pyrazole H-2), $8.86(\mathrm{~d}, 1 \mathrm{H}, J=5.0 \mathrm{~Hz}, \mathrm{H}-5), 10.54(\mathrm{~s}, 1 \mathrm{H}, \mathrm{NH})$ and $11.10 \mathrm{ppm}(\mathrm{s}, 1 \mathrm{H}, \mathrm{NH}) ; \mathrm{MS}(\mathrm{EI}): \mathrm{m} / \mathrm{z}(\%)$ $388\left(\mathrm{M}^{+}+1,9.0\right), 387\left(\mathrm{M}^{+}, 36.0\right), 360$ (17.8), 265 (100), 105 (87.2), 77 (.38.5). Anal. Calcd. for $\mathrm{C}_{20} \mathrm{H}_{17} \mathrm{~N}_{7} \mathrm{O}_{2}$ (387.40): C, 62.01; H, 4.42; N, 25.31. Found: C, 61.89; H, 4.54; N, 25.26.

\section{General procedure for the preparation of $17 a-c$}

Mixtures of 9a (1.15 g, 5mmol) and enaminone 16a-c (5 mmol) in pyridine $(20 \mathrm{~mL})$ were stirred at reflux for $5 \mathrm{~h}$, cooled to room temperature and poured into ice cold water. The aqueous solution was acidified with hydrochloric acid $(2 \mathrm{~N})$, forming asolid that was collected by filtration, washed with water and crystallized from the appropriate solvent.

(E)- $N$-(4,6-Dimethylpyrimidin-2-yl)-5-(3-oxo-3-phenylprop-1-enylamino)-1H-pyrazole-4-

carboxamide (17a). Recrystallized from DMF as pale brown crystals, $1.3 \mathrm{~g}(70 \%)$, mp $267{ }^{\circ} \mathrm{C}$; IR (IR (KBr): 3335, 3173, 3145 (3 NH), 1672,1631 cm-1 (2 CO); 1H NMR (DMSO-d6) : $\delta$ 2.41(s, 6H, 2CH3), 6.21(d, J = 8.0 Hz, 1H, prop-1-enylamino H-2), 6.98 (s, 1H, pyrimidine H-5), 7.49-7.98 (m, 6H, Ar-H and prop-1-enylamino H-1), 8.63 (s, 1H, pyrazole H-3), 10.54 (s, 1H, 
$\mathrm{NH}), 12.43(\mathrm{~d}, J=12.8 \mathrm{~Hz}, 1 \mathrm{H}, \mathrm{NH})$ and $12.95 \mathrm{ppm}(\mathrm{s}, 1 \mathrm{H}, \mathrm{NH})$; MS (EI): m/z (\%) $363\left(\mathrm{M}^{+}+1\right.$, 6.5), $362\left(\mathrm{M}^{+}, 21.0\right), 317$ (28.5), 223 (14.8), 222 (100). Anal. Calcd. for $\mathrm{C}_{19} \mathrm{H}_{18} \mathrm{~N}_{6} \mathrm{O}_{2}$ (362.39): C, 62.97; H, 5.01; N, 23.19. Found: C, 63.12; H, 4.95; N, 23.14.

(E)-N-(4,6-Dimethylpyrimidin-2-yl)-5-[3-oxo-3-(thiophen-2-yl)prop-1-enylamino]-1H-pyrazole-4carboxamide (17b). Recrystallized from DMF as yellow crystals, $1.2 \mathrm{~g}(66 \%), 279{ }^{\circ} \mathrm{C}$; IR (KBr): 3308, 3149, 3109 (3 NH), 1671,1626 cm ${ }^{-1}$ (2 CO); ${ }^{1} \mathrm{H}$ NMR (DMSO-d $): \delta 2.41\left(\mathrm{~s}, 6 \mathrm{H}, 2 \mathrm{CH}_{3}\right)$, 6.09 (d, $J=8.0 \mathrm{~Hz}, 1 \mathrm{H}$, prop-1-enylamino H-2), 6.99 (s, 1H, pyrimidine $\mathrm{H}-5), 7.20-7.87$ (m, 4H, Ar-H and prop-1-enylamino $\mathrm{H}-1), 8.61(\mathrm{~s}, 1 \mathrm{H}$, pyrazole $\mathrm{H}-3), 10.53(\mathrm{~s}, 1 \mathrm{H}, \mathrm{NH}), 12.21(\mathrm{~d}, J=$ $12.6 \mathrm{~Hz}, 1 \mathrm{H}, \mathrm{NH})$ and $12.93 \mathrm{ppm}(\mathrm{s}, 1 \mathrm{H}, \mathrm{NH}) ;{ }^{13} \mathrm{C}$ NMR $\left(\mathrm{DMSO}-d_{6}\right): \delta 94.53,102.19,115.60$, $128.58,129.96,131.19,132.97,142.19,146.18,157.39,161.13,162.32,167.60$ and $182.70 \mathrm{ppm}$ (Ar-C and 2CO); MS (EI): m/z (\%) $369\left(\mathrm{M}^{+}+1,3.8\right), 368\left(\mathrm{M}^{+}, 16.0\right), 350(14), 323$ (19.5), 229 (10.3), 228 (100). Anal. Calcd. for $\mathrm{C}_{17} \mathrm{H}_{16} \mathrm{~N}_{6} \mathrm{O}_{2} \mathrm{~S}$ (368.42): C, 55.42; H, 4.38; N, 22.81; S, 8.70. Found: C, 55.61; H, 4.42; N, 22.75; S, 8.59.

(E)-N-(4,6-Dimethylpyrimidin-2-yl)-5-[3-(6-methylpyridin-2-yl)3-oxoprop-1-enylamino]-1H-pyrazole4-carboxamide (17c). Recrystallized from DMF as pale yellow crystals, $1.35 \mathrm{~g} \mathrm{(73 \% ),} 299{ }^{\circ} \mathrm{C}$; IR (KBr): 3336, 3266, 3143 (3 NH), 1673,1631 cm ${ }^{-1}$ (2 CO); ${ }^{1} \mathrm{H}$ NMR (DMSO-d $): \delta 2.41(\mathrm{~s}, 6 \mathrm{H}$, $\left.2 \mathrm{CH}_{3}\right), 2.56\left(\mathrm{~s}, 3 \mathrm{H}, \mathrm{CH}_{3}\right), 6.73(\mathrm{~d}, J=8.0 \mathrm{~Hz}, 1 \mathrm{H}$, prop-1-enylamino $\mathrm{H}-2), 7.00(\mathrm{~s}, 1 \mathrm{H}$, pyrimidine $\mathrm{H}-5), 7.42-7.96$ (m, 4H, Ar-H and prop-1-enylamino $\mathrm{H}-1), 8.62(\mathrm{~s}, 1 \mathrm{H}$, pyrazole $\mathrm{H}-3)$, $10.55(\mathrm{~s}, 1 \mathrm{H}, \mathrm{NH}), 12.43$ (d, J=12.4 Hz, 1H, NH) and $12.97 \mathrm{ppm}(\mathrm{s}, 1 \mathrm{H}, \mathrm{NH}) ; \mathrm{MS}(\mathrm{EI}): \mathrm{m} / \mathrm{z}(\%)$ $378\left(\mathrm{M}^{+}+1,3.2\right), 377\left(\mathrm{M}^{+}, 12.4\right), 332$ (24.4), 236 (11.3), 237 (100). Anal. Calcd. for $\mathrm{C}_{19} \mathrm{H}_{19} \mathrm{~N}_{7} \mathrm{O}_{2}$ (377.41): C, 60.47; H, 5.07; N, 25.98. Found: C, 60.33; H, 4.98; N, 26.04.

\section{General procedure for the preparation of 18a-f}

Mixtures of 9a,b $(5 \mathrm{mmol})$ and enaminone 16a-c $(5 \mathrm{mmol})$ in pyridine $(20 \mathrm{~mL})$ were stirred at reflux overnight, or refluxing 13a-c $(5 \mathrm{mmol})$ in DMF containing anhydrous sodium acetate $(1 \mathrm{~g})$ for $6 \mathrm{~h}$. The reaction mixtures were cooled to room temperature and poured onto ice cold water. The aqueous solution was acidified with hydrochloric acid $(2 \mathrm{~N})$, forming a solid that was collected by filtration, washed with water and crystallized from the appropriate solvent.

$\mathbf{N}$-(4,6-Dimethylpyrimidin-2-yl)-7-phenylpyrazolo[1,5-a]pyrimidine-3-carboxamide (18a).

Recrystallized from DMF as buff crystals, $1.4 \mathrm{~g}(81 \%), \mathrm{mp} 270{ }^{\circ} \mathrm{C}$; IR (KBr): 3278 (NH), 1696 $\mathrm{cm}^{-1}(\mathrm{CO}) ;{ }^{1} \mathrm{H}$ NMR $\left(\mathrm{CDCl}_{3}\right): \delta 2.52\left(\mathrm{~s}, 6 \mathrm{H}, 2 \mathrm{CH}_{3}\right), 6.79(\mathrm{~s}, 1 \mathrm{H}$, pyrimidine $\mathrm{H}-5), 7.14(\mathrm{~d}, J=5.0$ $\mathrm{Hz}, 1 \mathrm{H}, \mathrm{H}-6), 7.60-8.07$ (m, 5H, Ar-H), 7.80 (d, 1H, J=5.0 Hz, H-5), 8.82 (s, 1H, pyrazole H2) and $10.67 \mathrm{ppm}(\mathrm{s}, 1 \mathrm{H}, \mathrm{NH}) ;{ }^{13} \mathrm{C} \mathrm{NMR}\left(\mathrm{CDCl}_{3}\right): \delta 24.15\left(2 \mathrm{CH}_{3}\right), 106.04,108.70,115.64$, $128.90,129.52,129.89,131.87,146.99,147.47,148.52,151.10,157.81,159.30,168.27$ (Ar-C and CO); MS (EI): m/z (\%) $345\left(\mathrm{M}^{+}+1,3.8\right), 344\left(\mathrm{M}^{+}, 12.5\right), 317$ (27.3), 223 (13.4), 222 (100). Anal. Calcd. for $\mathrm{C}_{19} \mathrm{H}_{16} \mathrm{~N}_{6} \mathrm{O}$ (344.38): C, 66.27; H, 4.68; N, 24.40. Found: C, 66.21; H, 4.75; N, 24.42 .

$\mathrm{N}$-(4,6-Dimethylpyrimidin-2-yl)-7-(thiophen-2-yl)pyrazolo[1,5-a]pyrimidine-3-carboxamide (18b). Recrystallized from DMF as yellow crystals, $1.5 \mathrm{~g}(86 \%), \mathrm{mp}$ above $300{ }^{\circ} \mathrm{C}$; IR (KBr): $3249(\mathrm{NH}), 1690 \mathrm{~cm}^{-1}(\mathrm{CO}) ;{ }^{1} \mathrm{H} \mathrm{NMR}\left(\mathrm{CDCl}_{3}\right): \delta 2.53\left(\mathrm{~s}, 6 \mathrm{H}, 2 \mathrm{CH}_{3}\right), 6.80(\mathrm{~s}, 1 \mathrm{H}$, pyrimidine $\mathrm{H}-$ 
5), 7.33 (t, $J=6.0 \mathrm{~Hz}, 1 \mathrm{H}$, thiophene H), 7.44 (d, $J=4.8 \mathrm{~Hz}, 1 \mathrm{H}, \mathrm{H}-6), 7.84$ (d, $J=6.0 \mathrm{~Hz}, 1 \mathrm{H}$, thiophene $\mathrm{H}), 8.44(\mathrm{~d}, J=6.0 \mathrm{~Hz}, 1 \mathrm{H}$, thiophene $\mathrm{H}), 8.75(\mathrm{~d}, 1 \mathrm{H}, J=4.8 \mathrm{~Hz}, \mathrm{H}-5), 8.91(\mathrm{~s}, 1 \mathrm{H}$, pyrazole $\mathrm{H}-2)$ and $10.70 \mathrm{ppm}(\mathrm{s}, 1 \mathrm{H}, \mathrm{NH}) ;{ }^{13} \mathrm{C} \mathrm{NMR}\left(\mathrm{CDCl}_{3}\right): \delta 24.12\left(2 \mathrm{CH}_{3}\right), 104.97,105.86$, $115.59,128.12,130.40,132.86,134.12,141.64,147.03,147.11,150.20,157.75,159.33,168.26$ (Ar-C and CO); MS (EI): m/z (\%) $351\left(\mathrm{M}^{+}+1,4.5\right), 350\left(\mathrm{M}^{+}, 17.25\right), 323$ (27.2), 229 (12.8), 228 (100). Anal. Calcd. for $\mathrm{C}_{17} \mathrm{H}_{14} \mathrm{~N}_{6} \mathrm{OS}$ (350.40): C, 58.27; H, 4.03; N, 23.98; S, 9.15. Found: C, 58.32; H, 3.98; N, 23.80; S, 9.06.

$\boldsymbol{N}$-(4,6-Dimethylpyrimidin-2-yl)-7-(6-methylpyridin-2-yl)pyrazolo[1,5-a]pyrimidine-3-carboxamide (18c). Recrystallized from DMF as pale yellow crystals, $1.4 \mathrm{~g}(77 \%), \mathrm{mp}$ above $300{ }^{\circ} \mathrm{C}$; IR (KBr): $3270(\mathrm{NH}), 1691 \mathrm{~cm}^{-1}(\mathrm{CO}) ;{ }^{1} \mathrm{H}$ NMR (DMSO-d $): \delta 2.53\left(\mathrm{~s}, 6 \mathrm{H}, 2 \mathrm{CH}_{3}\right), 2.70(\mathrm{~s}, 3 \mathrm{H}$, $\mathrm{CH}_{3}$ ), 6.80 (s, 1H, pyrimidine H-5), 7.39 (d, $\left.J=7.8 \mathrm{~Hz}, 1 \mathrm{H}, \mathrm{Ar}-\mathrm{H}\right), 7.87$ (t, $J=7.8 \mathrm{~Hz}, 1 \mathrm{H}$, Ar$\mathrm{H}), 8.00(\mathrm{~d}, J=4.8 \mathrm{~Hz}, 1 \mathrm{H}, \mathrm{H}-6), 7.88-8.92$ (m, 3H, 1 Ar-H, H-5 and pyrazole H-2) and 10.74 ppm (s, 1H, NH); ${ }^{13} \mathrm{C}$ NMR (DMSO-d $): \delta 24.12\left(2 \mathrm{CH}_{3}\right), 24.69\left(\mathrm{CH}_{3}\right), 105.89,109.26,115.64$, $123.81,125.93,137.05,146.04,146.42,147.22,147.29,151.17,157.72,159.31,159.32,168.30$ (Ar-C and CO); MS (EI): m/z (\%) $360\left(\mathrm{M}^{+}+1,3.0 \%\right), 359\left(\mathrm{M}^{+}, 9.5 \%\right.$ ), 332 (31.7), 238 (13.4), 237 (100). Anal. Calcd. for $\mathrm{C}_{19} \mathrm{H}_{17} \mathrm{~N}_{7} \mathrm{O}$ (359.39): C, 63.50; H, 4.77; N, 27.28. Found: C, 63.59; H, 4.64; N, 27.32.

$\boldsymbol{N}$-(2-Chloro-5-nitrophenyl)-7-phenylpyrazolo[1,5-a]pyrimidine-3-carboxamide (18d). Recrystallized from DMF as creamy white crystals, $1.35 \mathrm{~g}(69 \%)$, mp above $300^{\circ} \mathrm{C}$; $\mathrm{IR}(\mathrm{KBr}): 3270(\mathrm{NH}), 1674 \mathrm{~cm}^{-1}$ (CO); ${ }^{1} \mathrm{H}$ NMR (DMSO- $d_{6}$ ) at $110{ }^{\circ} \mathrm{C}: \delta$ 7.54-7.67 (m, 4H, Ar-H), 7.85 (d, J=9.0 Hz, 1H, Ar-H), 7.97 (d, $J=9.0 \mathrm{~Hz}, 1 \mathrm{H}, \mathrm{Ar}-\mathrm{H}), 8.18-8.98$ (m, 4H, H-6, H-5 and 2 Ar-H), 9.45 (s, 1H, pyrazole H-2) and $10.78 \mathrm{ppm}(\mathrm{s}, 1 \mathrm{H}, \mathrm{NH}) ;{ }^{13} \mathrm{C}$ NMR $(\mathrm{TFA}-\mathrm{d}): \delta 104.98,112.18,123.36,125.36,129.21$, $132.21,133.10,133.61,135.61,138.48,138.60,144.89,148.72,148.93,150.03,161.84$ and 163.94 (Ar-C and CO); MS (EI): m/z (\%) $394\left(\mathrm{M}^{+}+1,1.2\right) 393\left(\mathrm{M}^{+}, 5.2\right.$ ), 223 (16.9), 222 (100). Anal. Calcd. for $\mathrm{C}_{19} \mathrm{H}_{12} \mathrm{ClN}_{5} \mathrm{O}_{3}$ (393.79): C, 57.95; H, 3.07; Cl, 9.00; N, 17.78. Found: C, 58.06; $\mathrm{H}, 3.12 ; \mathrm{Cl}, 8.85 ; \mathrm{N}, 17.88$.

$\mathrm{N}$-(2-Chloro-5-nitrophenyl)-7-(thiophen-2-yl)pyrazolo[1,5-a]pyrimidine-3-carboxamide (18e). Recrystallized from DMF as pale yellow crystals, $1.2 \mathrm{~g}(60 \%)$, mp above $300{ }^{\circ} \mathrm{C}$; IR (KBr): $3265(\mathrm{NH}), 1674$ $\mathrm{cm}^{-1}(\mathrm{CO}) ;{ }^{1} \mathrm{H}$ NMR (DMSO- $d_{6}$ ) at $110{ }^{\circ} \mathrm{C}: \delta$ 7.42-8.91 (m, 8H, 6 Ar-H, H-6 and H-5), 9.46 (s, $1 \mathrm{H}$, pyrazole $\mathrm{H}-2)$ and $10.78 \mathrm{ppm}(\mathrm{s}, 1 \mathrm{H}, \mathrm{NH}) ;{ }^{13} \mathrm{C}$ NMR (TFA-d): $\delta 104.06,106.89,123.52$, $125.45,130.57,133.03,133.71,135.69,138.81,143.97,144.37,145.64,147.66,149.07,149.81$, 153.54, and 163.56 (Ar-C and CO); MS (EI): $\mathrm{m} / \mathrm{z}(\%) 400\left(\mathrm{M}^{+}+1,6.0\right), 399\left(\mathrm{M}^{+}, 23.5\right), 227$ (49.5), 228 (100), 200 (18.9). Anal. Calcd. for $\mathrm{C}_{17} \mathrm{H}_{10} \mathrm{ClN}_{5} \mathrm{O}_{3} \mathrm{~S}$ (399.82): C, 51.07; H, 2.52; Cl, 8.87; N, 17.52; S, 8.02. Found: C, 50.94; H, 2.60; Cl, 9.00 ; N, 17.66; S, 8.11.

$\mathbf{N}$-(2-Chloro-5-nitrophenyl)-7-(6-methylpyridin-2-yl)pyrazolo[1,5-a]pyrimidine-3-carboxamide (18f). Recrystallized from DMF as creamy white crystals, $1.35 \mathrm{~g}(69 \%), \mathrm{mp} 297-299{ }^{\circ} \mathrm{C}$; IR (KBr): $3280(\mathrm{NH})$, $1695 \mathrm{~cm}^{-1}(\mathrm{CO}) ;{ }^{1} \mathrm{H}$ NMR (DMSO-d $)_{6}$ ) at $110{ }^{\circ} \mathrm{C}: \delta 2.66\left(\mathrm{~s}, 3 \mathrm{H}, \mathrm{CH}_{3}\right), 7.56-9.06(\mathrm{~m}, 8 \mathrm{H}, 6 \mathrm{Ar}-\mathrm{H}, \mathrm{H}-6$ and $\mathrm{H}-5), 9.48$ (s, $1 \mathrm{H}$, pyrazole $\mathrm{H}-2)$ and $10.83 \mathrm{ppm}(\mathrm{s}, 1 \mathrm{H}, \mathrm{NH}) ;{ }^{13} \mathrm{C}$ NMR (TFA-d): $\delta 22.05$ $\left(\mathrm{CH}_{3}\right), 108.37,113.16,119.99,123.14,126.56,132.95,133.30,134.80,137.04,137.36,144.04$, 148.94, 149.22, 149.79, 150.24, 155.71, 158.41 and 163.57 (Ar-C and CO); MS (EI): m/z (\%) 47 
B09 $\left(\mathrm{M}^{+}+1,1.3\right), 408\left(\mathrm{M}^{+}, 4.5\right), 238$ (14.5), 237 (100). Anal. Calcd. for $\mathrm{C}_{19} \mathrm{H}_{13} \mathrm{ClN}_{6} \mathrm{O}_{3}(408.81)$ : C, 55.82; H, 3.21; Cl, 8.67; N, 20.56. Found: C, 55.75; H, 3.32; Cl, 8.80; N, 20.49.

\section{General procedure for the preparation of 19 and 21}

A mixture of 9b (1.4 g, $5 \mathrm{mmol})$ and DMFDMA (0.6 g, $5 \mathrm{mmol})$ in case of 19 or $(1.3 \mathrm{~g}, 11$ $\mathrm{mmol})$ in case of 21, in DMF (20 mL) was stirred at reflux for $6 \mathrm{~h}$, cooled to room temperature and poured onto ice cold water. The formed crude solid was collected by filtration, washed with water and crystallized from the appropriate solvent.

(E)-N-(2-Chloro-5-nitrophenyl)-5-[(dimethylamino)methyleneamino]-1H-pyrazolo-4-carboxamide (19). Recrystallized from EtOH as yellow crystals,.25 g (75 \%), mp $237{ }^{\circ} \mathrm{C}$; IR (KBr): 3426, $3213(2 \mathrm{NH}), 1669$ $\mathrm{cm}^{-1}(\mathrm{CO}) .{ }^{1} \mathrm{H}$ NMR (DMSO-d $): \delta 3.08\left(\mathrm{~s}, 3 \mathrm{H}, \mathrm{CH}_{3}\right), 3.11\left(\mathrm{~s}, 3 \mathrm{H}, \mathrm{CH}_{3}\right), 7.80(\mathrm{~d}, J=8.8 \mathrm{~Hz}, 1 \mathrm{H}$, Ar-H), 7.92 (d, $J=8.8 \mathrm{~Hz}, 1 \mathrm{H}, \mathrm{Ar}-\mathrm{H}), 8.12$ (s, 1H, Ar-H), 8.28 (s, 1H, imino CH), 9.27 (s, 1H, pyrazole $\mathrm{H}-3) 10.77(\mathrm{br}, 1 \mathrm{H}, \mathrm{NH})$ and $12.71 \mathrm{ppm}(\mathrm{s}, 1 \mathrm{H}, \mathrm{NH}) ;{ }^{13} \mathrm{C} \mathrm{NMR}\left(\mathrm{CDCl}_{3}\right): \delta 34.66$ $\left(\mathrm{CH}_{3}\right), 40.34\left(\mathrm{CH}_{3}\right), 106.63,116.49,118.37,128.61,130.24,133.04,136.43,146.35,155.32$, 156.09 and $161.75 \mathrm{ppm}$ (carbons and CO); MS (EI): m/z (\%) $337\left(\mathrm{M}^{+}+1,7.8\right), 336\left(\mathrm{M}^{+}, 25.0\right)$, 256 (20.8), 210 (12.4), 165 (100) 120 (24.6). Anal. Calcd. for $\mathrm{C}_{13} \mathrm{H}_{13} \mathrm{ClN}_{6} \mathrm{O}_{3}$ (336.74): C, 46.37; H, 3.89; Cl, 10.53; N, 24.96. Found: C, 46.44; H, 3.78; Cl, 10.66; N, 25.06.

(E)-N-(2-Chloro-5-nitrophenyl)-5-[(dimethylamino)methyleneamino]-1-methyl-1H-pyrazolo-4-

carboxamide (21). Recrystallized from EtOH/DMF as yellow crystals, $1.6 \mathrm{~g} \mathrm{(92 \% ),} \mathrm{mp} 234{ }^{\circ} \mathrm{C}$; IR (KBr): $3121(\mathrm{NH}), 1691 \mathrm{~cm}^{-1}(\mathrm{CO}) .{ }^{1} \mathrm{H}$ NMR (DMSO-d $): \delta 3.13\left(\mathrm{~s}, 3 \mathrm{H}, \mathrm{CH}_{3}\right), 3.16(\mathrm{~s}, 3 \mathrm{H}$, $\left.\mathrm{CH}_{3}\right), 3.84$ (s, 3H, $\left.\mathrm{CH}_{3}\right), 7.53$ (d, $\left.J=8.0 \mathrm{~Hz}, 1 \mathrm{H}, \mathrm{Ar}-\mathrm{H}\right), 7.86-7.90$ (m, 2H, Ar-H), 8.26 (s, 1H, imino $\mathrm{CH}$ ), $9.38(\mathrm{~s}, 1 \mathrm{H}$, pyrazole $\mathrm{H}-3)$ and $10.65 \mathrm{ppm}(\mathrm{s}, 1 \mathrm{H}, \mathrm{NH}) ;{ }^{13} \mathrm{C}$ NMR (DMSO- $\left.d_{6}\right): \delta$ $35.25\left(\mathrm{CH}_{3}\right), 39.38\left(\mathrm{CH}_{3}\right), 40.99\left(\mathrm{CH}_{3}\right), 108.39,118.09,118.30,129.49,129.80,134.75,136.89$, 146.96, 154.93, 156.25 and $162.00 \mathrm{ppm}$ (carbons and CO); $\mathrm{MS}(\mathrm{EI}): \mathrm{m} / \mathrm{z}(\%) 351\left(\mathrm{M}^{+}+1,6.5\right)$, $350\left(\mathrm{M}^{+}, 21.0\right), 179$ (100), 134 (36.4). Anal. Calcd. for $\mathrm{C}_{14} \mathrm{H}_{15} \mathrm{ClN}_{6} \mathrm{O}_{3}$ (350.77): C, 47.94; $\mathrm{H}$, 4.31; Cl, 10.11; N, 23.96. Found: C, 48.10; H, 4.36; Cl, 9.98; N, 24.03.

\section{General procedure for the preparation of 22a-d}

A cold solution of the aryldiazonium salts $(10 \mathrm{mmol})$ was prepared by adding a solution of sodium nitrite (1.4 g dissolved in $10 \mathrm{~mL}$ water) to a cold solution of arylamine hydrochloride (10 $\mathrm{mmol}$ in $6 \mathrm{~mL}, 6 \mathrm{M} \mathrm{HCl}$ ) with stirring. The resulting solution of aryldiazonium salts were then added to a cold solution of 3-oxoalkanonitriles 5a-c in ethanol $(50 \mathrm{~mL})$ in the presence of sodium acetate trihydrate $(4.2 \mathrm{~g}, 30 \mathrm{mmol})$. The mixture was stirred at room temperature for $1 \mathrm{~h}$ and the solid was collected by filtration, washed with water and crystallized from the appropriate solvent.

(E)-2-Cyano- $N$-(4,6-dimethylpyrimidin-2-yl)-2-(2-phenylhydrazono)acetamide (22a). Recrystallized from EtOH as yellow crystals, $2.45 \mathrm{~g}(83 \%), \mathrm{mp} 2202{ }^{\circ} \mathrm{C}$; IR (KBr): 3376, $3186(2 \mathrm{NH}), 2218$ $(\mathrm{CN}), 1700 \mathrm{~cm}^{-1}(\mathrm{CO}) ;{ }^{1} \mathrm{H}$ NMR (DMSO-d 6 ): $\delta 3.37$ (s, 6H, 2CH3), 7.00 (s, 1H, pyrimidine H-5), 7.12 (t, $J=8.0 \mathrm{~Hz}, 1 \mathrm{H}, \mathrm{Ar}-\mathrm{H}$ ), 7.37 (t, $J=8.0 \mathrm{~Hz}, 2 \mathrm{H}, \mathrm{Ar}-\mathrm{H}$ ), 7.65 (d, J=8.0 Hz, 2H, Ar-H ), $10.23(\mathrm{~s}, 1 \mathrm{H}, \mathrm{NH})$ and $11.96 \mathrm{ppm}(\mathrm{s}, 1 \mathrm{H}, \mathrm{NH}) ; \mathrm{MS}(\mathrm{EI}): \mathrm{m} / \mathrm{z}(\%) 295\left(\mathrm{M}^{+}+1,14.3\right), 294\left(\mathrm{M}^{+}\right.$, 
87.9), 189 (68.4), 188 (53), 150 (100), 123 (19.5). Anal. Calcd. for $\mathrm{C}_{15} \mathrm{H}_{14} \mathrm{~N}_{6} \mathrm{O}$ (294.32): C, 61.22; H, 4.79; N, 28.55. Found: C, 61.40; H, 4.65; N, 28.42.

(E)-2-[2-(4-Chlorophenyl)hydrazono]-2-cyano- $\mathbf{N}$-(4,6-dimethylpyrimidin-2-yl)acetamide (22b). Recrystallized from EtOH as yellow crystals, $2.6 \mathrm{~g} \mathrm{(79 \% ),} \mathrm{mp} 224{ }^{\circ} \mathrm{C}$; IR (KBr): 3393 , $3177(2 \mathrm{NH}), 2214(\mathrm{CN}), 1693 \mathrm{~cm}^{-1}(\mathrm{CO}) ;{ }^{1} \mathrm{H}$ NMR (DMSO-d $): \delta 3.36\left(\mathrm{~s}, 6 \mathrm{H}, 2 \mathrm{CH}_{3}\right), 7.00(\mathrm{~s}$, 1H, pyrimidine H-5), 7.39 (d, $J=8.8 \mathrm{~Hz}, 2 \mathrm{H}, \mathrm{Ar}-\mathrm{H}$ ), 7.63 (d, $J=8.8 \mathrm{~Hz}, 2 \mathrm{H}, \mathrm{Ar}-\mathrm{H}$ ), 10.12 (s, $1 \mathrm{H}, \mathrm{NH})$ and $13.04 \mathrm{ppm}(\mathrm{br}, 1 \mathrm{H}, \mathrm{NH}) ;{ }^{13} \mathrm{C} \mathrm{NMR}\left(\mathrm{DMSO}-d_{6}\right): \delta 23.86\left(2 \mathrm{CH}_{3}\right), 108.37,111.79$, $117.08(\mathrm{CN}), 118.75,129.24,129.79,141.79,156.90,160.07$ and $168.87 \mathrm{ppm}$ (Ar-C and CO); MS (EI): m/z (\%) $330\left(\mathrm{M}^{+}+2,22.4\right), 329\left(\mathrm{M}^{+}+1,13.7\right), 328\left(\mathrm{M}^{+}, 67.5\right), 189$ (48.6), 188 (62.8), 150 (100), 123 (21.2). Anal. Calcd. for $\mathrm{C}_{15} \mathrm{H}_{13} \mathrm{ClN}_{6} \mathrm{O}$ (328.76): C, 54.80; H, 3.99; Cl, 10.78; N, 25.56. Found: C, 54.65; H, 4.11; Cl, 10.93; N, 25.37.

(E)- $\mathrm{N}$-(2-Chloro-5-nitrophenyl)-2-cyano-2-(2-phenylhydrazono)acetamide (22c). Recrystallized from EtOH/DMF as orange crystals, $3.0 \mathrm{~g}(89 \%), \mathrm{mp} 268-270{ }^{\circ} \mathrm{C}$; IR (KBr): 3359, $3227(2 \mathrm{NH})$, $2212(\mathrm{CN}), 1675 \mathrm{~cm}^{-1}(\mathrm{CO}) ;{ }^{1} \mathrm{H}$ NMR (DMSO-d $): \delta 7.14(\mathrm{t}, J=8.0 \mathrm{~Hz}, 1 \mathrm{H}, \mathrm{Ar}-\mathrm{H}), 7.39(\mathrm{t}, J=$ $8.0 \mathrm{~Hz}, 2 \mathrm{H}, \mathrm{Ar}-\mathrm{H}$ ), 7.57 (d, J=8.0 Hz, 2H, Ar-H ), 7.82 (d, J=7.6 Hz, 1H, Ar-H ), 8.00 (d, $J=$ $7.6 \mathrm{~Hz}, 1 \mathrm{H}, \mathrm{Ar}-\mathrm{H}), 8.85$ (s, 1H, Ar-H), $9.68(\mathrm{~s}, 1 \mathrm{H}, \mathrm{NH})$ and $12.31 \mathrm{ppm}(\mathrm{br}, 1 \mathrm{H}, \mathrm{NH}) ;{ }^{13} \mathrm{C}$ NMR (DMSO- $d_{6}$ ): 106.07, 110.82, 116.09 (CN), 117.72, 120.33, 124.87, 129.42, 130.60, 132.01, 135.14, 141.77, 146.43 and 159.62 ppm (Ar-C and CO); MS (EI): m/z (\%) $345\left(\mathrm{M}^{+}+2,28.6\right)$, $344\left(\mathrm{M}^{+}+1,15.3\right), 343\left(\mathrm{M}^{+}, 97.2\right), 172$ (100), 143 (24.6). Anal. Calcd. for $\mathrm{C}_{15} \mathrm{H}_{10} \mathrm{ClN}_{5} \mathrm{O}_{3}$ (343.73): C, 52.42; H, 2.93; Cl, 10.31; N, 20.37. Found: C, 52.54; H, 3.04; Cl, 10.24; N, 20.48.

\section{Crystallographic analysis for 22c}

Crystals were mounted on a glass fiber. All measurements were performed on an ENRAF NONIUS FR 590. The data were collected at $25{ }^{\circ} \mathrm{C}$ using the $\omega$ scanning technique to a maximum of a $2 \theta$ of $24.108^{\circ}$. The structure was solved by direct methods using SIR 92 and refined by full-matrix least squares. Non-hydrogen atoms were refined anisotropically. Hydrogen atoms were located geometrically and were refined isotropically.

\section{Crystal data}

$\mathrm{C}_{15} \mathrm{H}_{10} \mathrm{ClN}_{5} \mathrm{O}_{3}, \mathrm{M}_{\mathrm{r}}=343.730$, monoclinic, $\mathrm{a}=12.6511$ (4) $\AA, \mathrm{b}=8.2357$ (3) $\AA, \mathrm{c}=16.9705$ (8) $\AA$, $\mathrm{V}=1532.88(10) \AA^{3}, \alpha=\gamma=90.00^{\circ}, \beta=12$. (18) $\mathrm{x} 10^{1 \circ}$, space group: $\mathrm{P} 2{ }_{1} / \mathrm{c}, \mathrm{D}_{\mathrm{x}}=1.489 \mathrm{Mg} \mathrm{m}^{-3}$ reflection 9737 measured, $\theta_{\max }=27.50^{\circ}, \omega \mathrm{R}$ factor $=0.075$. Figure 1 illustrates the structure as determined. Full data can be obtained on request from the CCDC. ${ }^{18}$

(E)-2-Cyano- $\boldsymbol{N}$-(2-fluoro-5-nitrophenyl)-2-(2-phenylhydrazono)acetamide (22d). Recrystallized from EtOH / Ddioxan as yellow crystals, $2.8 \mathrm{~g}(85 \%)$, mp $235{ }^{\circ} \mathrm{C}$; IR (KBr): 3389, 3233 (2 $\mathrm{NH}), 2214(\mathrm{CN}), 1682 \mathrm{~cm}^{-1}(\mathrm{CO}) ;{ }^{1} \mathrm{H}$ NMR $\left(\mathrm{DMSO}_{6}\right): \delta 7.16(\mathrm{t}, J=8.0 \mathrm{~Hz}, 1 \mathrm{H}, \mathrm{Ar}-\mathrm{H}), 7.41$ $(\mathrm{t}, J=8.0 \mathrm{~Hz}, 2 \mathrm{H}, \mathrm{Ar}-\mathrm{H}), 7.61-8.63(\mathrm{~m}, 5 \mathrm{H}, \mathrm{Ar}-\mathrm{H}), 10.05(\mathrm{~s}, 1 \mathrm{H}, \mathrm{NH})$ and $12.20 \mathrm{ppm}(\mathrm{s}, 1 \mathrm{H}$, $\mathrm{NH}) ;{ }^{13} \mathrm{C}$ NMR (DMSO-d $)$ : 106.45, 111.11, $116.32(\mathrm{CN}), 117.24,121.36,122.39,124.74$, 126.38, 129.29, 141.92, 143.69, 157.58 and 160.06 ppm (Ar-C and CO); MS (EI): m/z (\%) 328 
$\left(\mathrm{M}^{+}+1,22.5\right), 327\left(\mathrm{M}^{+}, 100\right), 172$ (26.5), 156 (43), 143 (39.3). Anal. Calcd. for $\mathrm{C}_{15} \mathrm{H}_{10} \mathrm{FN}_{5} \mathrm{O}_{3}$ (327.28): C, 55.05; H, 3.08; N, 21.40. Found: C, 54.89; H, 2.99; N, 21.52.

\section{General method for the preparation of 24 and 25}

A mixture of arylhydrazononitriles 22a,c $(5 \mathrm{mmol})$, and hydroxylamine hydrochloride $(0.5 \mathrm{~g}, 7.5$ mmol) was stirred at reflux in DMF $(20 \mathrm{~mL})$ in presence of anhydrous sodium acetate $(1 \mathrm{~g})$ for 5 $\mathrm{h}$. Then, the reaction mixture was a cooled to room temperature and poured onto ice cold water. The formed solid was collected by filtration washed with water and crystallized from EtOH/DMF mixture as pale brown crystals.

5-Amino- $\mathbf{N}$-(2-chloro-5-nitrophenyl)-2-phenyl-2H-1,2,3-triazole-4-carboxamide (24). Yield: $1.2 \mathrm{~g}\left(68 \%\right.$ ); mp: 193-195 ${ }^{\circ} \mathrm{C}$; IR (KBr): 3436, 3374, $3164\left(\mathrm{NH}_{2}\right.$ and $\left.\mathrm{NH}\right), 1684 \mathrm{~cm}^{-1}(\mathrm{CO}) ;{ }^{1} \mathrm{H}$ MNR (DMSO- $\left.d_{6}\right): \delta$ $6.21\left(\mathrm{~s}, 2 \mathrm{H}, \mathrm{NH}_{2}\right), 7.41-8.91(\mathrm{~m}, 8 \mathrm{H}, \mathrm{Ar}-\mathrm{H}), 9.89$ (s, 1H, NH); MS (EI): m/z (\%) $360\left(\mathrm{M}^{+}+2,68.3\right), 359\left(\mathrm{M}^{+}+1\right.$, 55.8), 368 ( $\mathrm{M}^{+}$, 86.8), 323 (47.2), 187 (100), 180 (38.1), 77 (87). Anal. Calcd for $\mathrm{C}_{15} \mathrm{H}_{11} \mathrm{ClN}_{6} \mathrm{O}_{3}$ (358.75): C, 50.22; H, 3.09; Cl, 9.88; N, 23.43. Found: C, 50.38; H, 2.89; Cl, 9.74; N, 23.60.

(E)-3-Amino-4-(2-phenylhydrazono)-4H-isoxazol-5-one (25). Yield: $0.7 \mathrm{~g} \mathrm{(70 \% );} \mathrm{mp:} 198{ }^{\circ} \mathrm{C}$; IR (KBr): 3420, 3294, $\left.3221\left(\mathrm{NH} 2 \text { and NH), } 1711 \mathrm{~cm}^{-1}(\mathrm{CO}) ;{ }^{1} \mathrm{H} \text { MNR (DMSO-d }\right)_{6}\right): \delta 6.40(\mathrm{~s}$, 2H, $\mathrm{NH}_{2}$ ), 7.19 (t, $J=8.0 \mathrm{~Hz}, 1 \mathrm{H}, \mathrm{Ar}-\mathrm{H}$ ), 7.40 (t, $J=8.0 \mathrm{~Hz}, 2 \mathrm{H}, \mathrm{Ar}-\mathrm{H}$ ), 7.60 (d, J=8.0 Hz, $2 \mathrm{H}, \mathrm{Ar}-\mathrm{H}$ ), 12.41 (br, $1 \mathrm{H}, \mathrm{NH}) ;{ }^{13} \mathrm{C}$ NMR (DMSO-d $)$ ): 115.91, 116.36, 125.40, 129.30, 141.79, 159.05 and $162.37 \mathrm{ppm}$ (Ar and CO); MS (EI): m/z (\%) $205\left(\mathrm{M}^{+}+1,16.3\right), 204\left(\mathrm{M}^{+}, 93.9\right), 186$ (16.3), 91 (100). Anal. Calcd. for $\mathrm{C}_{9} \mathrm{H}_{8} \mathrm{~N}_{4} \mathrm{O}_{2}$ (204.19): C, 52.94; H, 3.95; N, 27.67. Found: C, 53.06; H, 3.86; N, 27.52.

Note: The biological activities of the new zaleplon analogues prepared in this study are now under investigation and the results will be the subject of a further communication.

\section{Acknowledgements}

The support for this work was from University of Kuwait through research grant (SC03/07). The facilities of Analab/SAF (GS01/01), (GS01/05) and (GS01/03) are gratefully acknowledged. The authors are grateful to Prof. Dr. M. H. Elnagdi for his support and for reading the manuscript in its original form.

\section{References}

1. Petroski, R. E.; Pomeroy, J. E.; Das, R.; Bowman, H.; Yang, W.; Chen, A. P.; Foster, A. C. J. Pharmacol. Exp. Ther. 2006, 317, 369.

2. George, C. F. P. Lancet 2001, 358, 1623.

3. Drover, D.; Lemmens, H.; Naidu,S.; Cevallos, W.; Darwish, M.; Stanski, D. Clinical 
Therapeutics 2000, 22, 1443.

4. Weitzel, K. W.; Wickman, J. M.; Augustin, S. G.; Strom, J. G. Clinical Therapeutics 2000, 22, 1254.

5. Ming, L.; Wei-Si, G.; Li-Rong, W.; Bo, Q. Jiegou Нuaxue 2006, 25, 108.

6. Mirza, N. R.; Rodgers, R. J.; Mathiasen, L. S. J. Pharmacol. Exp. Ther. 2006, 316, 1291.

7. Ibrahim, H. M.; Makhseed, S.; Abdel-Motaleb, R. M.; Makhloof, A. A.; Elnagdi, M. H. Heterocycles 2007, 71, 1951.

8. Khalil, K. D.; Al-Matar, H. M.; Al-Dorri, D. M.; Elnagdi, M. H. Tetrahedron 2009, 65, 9421.

9. McFadden, H.G.; Huppatz, J.L.; Halladay, P.K. Austral. J. Chem. 1993, 46, 873.

10. Jianguo, S.; Peiyu, W.; Ming, Z.; Qi, Z. Yangzhou, Daxue Xuebao, Ziran Kexueban 1998, 1, 17.

11. Abdel-Motaleb, R. M.; Makhloof, A. A.; Ibrahim, H. M.; Elnagdi, M. H. J. Heterocycl. Chem. 2007, 44, 109.

12. Slatt, J.; Romero, I.; Bergman, J. Synthesis 2004, 2760.

13. Aziz, S. I.; Anwar, H. F.; Felita, D. H.; Elnagdi, M. H. J. Heterocycl. Chem. 2007, 44, 725.

14. Kenawi, I. M.; Elnagdi, M. H. Spectrochim. Acta Molecul. Biomolecul. Spectroscopy 2006, $65 A, 805$.

15. Ghozlan, S. A. S.; Abdelhamid, I. A.; Ibrahim, H. M.; Elnagdi, M. H. Arkivoc 2006, (xv), 53.

16. Al-Matar, H.M.; Riyadh, S.M.; Elnagdi, M. H., Arkivoc 2007,(xiii), 53.

17. Elnagdi, M. H.; Elmoghayer, M. R. H.; Hafez, E. A. A.; Alnima, H. H. J. Org. Chem. 1975, 40, 2604.

18. Crystal data for 22c (ref. CCDC 759425) can be obtained on request from the director, Cambridge Crystallographic Data Center, 12 Union Road, Cambrige CB2 1EW, UK. 